\title{
EDITORIAL
}

\section{CD4/CD8 ratios in bronchoalveolar lavage fluid: of value for diagnosing sarcoidosis?}

\author{
U. Costabel
}

Bronchoalveolar lavage (BAL) was originally developed as a research tool for investigation of the immunopathogenesis of interstitial and airway disorders. Quickly, the clinical potential of this technique was recognized, and today BAL is considered as a standard procedure in the diagnostic work-up of patients with all kinds of diffuse lung disease - at least in Germany and other European countries $[1,2]$

Initially, BAL was thought to provide complimentary information to histopathology but lately the question has arisen whether the method might even be able to replace biopsy in the evaluation of interstitial lung diseases. Clearly, lavage has some advantages over biopsy procedures. It is safe, noninvasive, and associated with virtually no morbidity, even in severely thrombocytopenic patients. Lethal complications directly attributable to BAL have not been reported. Furthermore, lavage samples a much larger area of the lungs than can be obtained by the small fragments of transbronchial biopsy or by open biopsy specimens. Therefore it presumably gives a better representative average picture of the inflammatory and immune changes in the lung than biopsies.

Transbronchial biopsy carries a minor risk of lethal complications due to massive bleeding or pneumothorax which ranges $0.1-0.2 \%[3,4]$. Open lung biopsy is an invasive procedure associated with morbidity, several days of hospital stay, and mortality rates ranging $1.8-21 \%$ in patients with chronic infiltrative lung disease [3,5], and up to $38 \%$ in those presenting with acute respiratory failure [6]. The enthusiasm for video-assisted thoracic surgery (VATS) as a bioptic approach for the assessment of interstitial lung disease is still increasing. Having a comparable diagnostic accuracy as open biopsy, mortality rates are lower, but a figure of $6 \%$ has been reported [5]. The advantages of lower morbidity, reduced time necessary for chest tube drainage and shorter duration of hospital stay as reported in some studies $[5,7,8]$ are counterbalanced by higher operation room costs [9]. RAVINI et al. [10] conclude in a paper to be published in the next issue of the European Respiratory Journal, including a number of pat-ients with sarcoidosis, that VATS is the best option when a surgical procedure is required for histological confirmation of diffuse lung disease after less invasive methods have failed.

In which settings can lavage really replace biopsy? Obviously, where BAL has high sensitivity and specificity, this obviates the need for biopsy for certain diseases, such as: the demonstration of adenocarcinoma cells typical for bronchoalveolar carcinoma or lymphangitis carcinomato-

Correspondence: U. Costabel, Ruhrlandklinik, Dept Pneumology/Allergology, Tueschener Weg 40, D-45239 Essen, Germany. sis; the finding of iron-laden macrophages with or without free red blood cells as in the alveolar haemorrhage syndromes; or the surprising aspect of a "milky" fluid return in the bronchoscopy room with Periodic acid-Schiff positive oval bodies and amorphous debris when the "milk" is seen under the microscope, all very characteristic signs of pulmonary alveolar proteinosis $[1,2]$. In the setting of pulmonary infection in an immunocompromised patient, lavage has probably achieved the greatest practical value. In patients infected with the human immunodeficiency virus (HIV), BAL is usually the only bronchoscopic procedure which is needed, and it is not necessary to perform transbronchial biopsy in parallel. The sensitivity of BAL for the demonstration of pneumocystis carinii exceeds $95 \%$ [1].

In other settings, things are not so easy. Most patients with noninfectious immunological interstitial lung disease show nonspecific cellular profiles. The list of diseases with a lymphocytic, a neutrophilic, an eosinophilic, or a mixed cellular pattern is long. In these patients it is important to keep in mind that lavage should not be used as isolated tool for making a diagnosis, but should always be interpreted in the context of disease history, clinical, laboratory and radiological findings. Clinical signs and highresolution computed tomography, both assessed by an experienced investigator, are often highly suggestive of a specific diagnosis, and a lavage finding being compatible with the suspected diagnosis may then be sufficient for disease confirmation. On the other hand, if the BAL pattern is not consistent, e.g. an isolated and marked increase in lymphocytes in a patient with clinical and radiological findings suggestive of idiopathic pulmonary fibrosis, further diagnostic work-up is clearly necessary.

What is the situation in sarcoidosis? It is evident that the increase in BAL lymphocytes is absolutely nonspecific, as many granulomatous and other interstitial lung disorders show a similar magnitude and range of lymphocyte percentages. Activated CD4+ helper/inducer Tcells are considered as the hallmark of early alveolitis in the initial phase of sarcoidosis [11]. In this respect, the value of the CD4/CD8 ratio of BAL lymphocytes for differentiating sarcoidosis from other causes of interstitial lung diseases has been examined by several authors [1214]. Three independent groups of investigators found almost identical sensitivity and specificity values of elevated CD4/CD8 ratios for diagnosing sarcoidosis. COSTABEL et al. [12] reported that a ratio of 3.5 or greater had a sensitivity of $52 \%$ and specificity of $94 \%$ in 117 consecutive patients with biopsy-proven sarcoidosis. WINTERBAUER et al. [13] described that a ratio of 4.0 or greater disting-uished 27 patients with sarcoidosis from 28 patients with other interstitial lung diseases with a sensitivity of $59 \%$ and a 
specificity of 96\%. Recently, THомеER and DEMEDTs [14] compared 42 patients with sarcoidosis and 90 pat-ients with other forms of diffuse lung disease and found that a CD4/ CD8 ratio of greater than 4.0 had a sensitivity of $55 \%$ and a specificity of $94 \%$. These three studies reached similar conclusions: in patients with a clinical picture typical of sarcoidosis, an elevated CD4/CD8 ratio in BAL may prove diagnostic of sarcoidosis and obviate the need for confirmation by additional biopsy. It is im-portant to note that in the study of WinTERBAUER et al. [13], transbronchial biopsy had a specificity of $89 \%$ for the distinction between sarcoidosis and other forms of diffuse lung disease, and was thus not better than the CD4/CD8 ratio in this respect.

A paper published in this issue of the Journal questions the clinical usefulness of the CD4/CD8 ratio based on the observation that this ratio is highly variable [15]. These authors found that only $42 \%$ in a population of 86 patients with biopsy-proven sarcoidosis had a ratio greater than 4.0 , and that $12 \%$ had an inversed ratio below 1.0. Why the sensitivity of the ratio was lower in this study than in the previous ones (42 versus 52-59\%) remains unclear. Different methologies or patient populations may be the reason. That the CD4/CD8 ratio may be decreased in patients in sarcoidosis, is not a new finding and has been seen before at a similar frequency of $10 \%[12,14]$. Thus, a low ratio cannot be used to exclude the diagnosis of sarcoidosis. Kantrow et al. [15] argue that they would not follow the potential clinical approach to proceed with BAL and cellular analysis without biopsy using a CD4/CD8 ratio greater than 4.0 to confirm the diagnosis of sarcoidosis, since in their population such an approach would have resulted in a nondiagnostic BAL (without biopsy) followed by a repeat bronchoscopy with biopsy in more than half of the cases.

I would see it the other way round. If I perform only BAL as a first step, the diagnosis can be confirmed in 50$60 \%$ of patients with sarcoidosis (this is the sensitivity of the CD4/CD8 ratio in the three above-mentioned studies). This would be a very safe approach for these patients, without any risk of major morbidity or the potential lethal complications discussed above for the various techniques. Only $40-50 \%$ of patients would then have to undergo the more invasive and risky biopsy procedures. Moreover, performance of this approach would also be feasible for practising pulmonologists in nonhospital facilities, as frequently done in Germany. Even if other physicians feel that transbronchial biopsy is a relatively safe technique, associated with little morbidity and almost no risk of lethal complications, the addition of BAL to this procedure at the same bronchoscopy will increase the diagnostic yield, as shown in this issue in the article by LeONARD et al. [16]. These authors used three bronchoscopic techniques, lavage, transbronchial needle aspiration, and transbronchial lung biopsy, and clearly showed an enhanced diagnostic yield, little added cost and no additional complications. The limitations of this study are, however, the very small number of only 13 patients. This number is surely too low for detection of differences in complication rates. In this series, the combination of the three techniques gave a diagnostic yield of $100 \%$ for the diagnosis of sarcoidosis.

In summary, the present evidence from literature suggests that bronchoalveolar lavage $\mathrm{CD} 4 / \mathrm{CD} 8$ ratios may be of diagnostic value in sarcoidosis, obviating the need of biopsy in $40-60 \%$ of patients. It may depend on the individual facilities, knowledge and skill of the investigators involved in the various diagnostic techniques, whether bronchoalveolar lavage should be considered as the only technique at the first bronchoscopy, or whether several techniques including lavage should be applied together to increase the diagnostic yield to an optimum at the first step.

\section{References}

1. Klech H, Hutter C, Costabel U, eds. Clinical guidelines and indications for bronchoalveolar lavage (BAL). Eur Respir Rev 1992; 2: 47-127.

2. Costabel U. Atlas of bronchoalveolar lavage. London, Chapman and Hall, 1998.

3. Wall CP, Gaensler EA, Carrington CB, Hayes JA. Comparison of transbronchial and open biopsies in chronic infiltrative lung diseases. Am Rev Respir Dis 1981; 123: 280-285.

4. Simpson FG, Arnold AG, Purvis A, Belfield PW, Muers MF, Cooke NJ. Postal survey of bronchoscopic practice by physicians in the United Kingdom. Thorax 1986; 41: 311-317.

5. Ferson PF, Landreneau RJ, Dowling RD, et al. Comparison of open versus thoracoscopic lung biopsy for diffuse infiltrative pulmonary disease. J Thorac Cardiovasc Surg 1993; 106: 194-199.

6. Bensard DD, McIntyre RC, Waring BJ, Simon JS. Comparison of video thoracoscopic lung biopsy to open lung biopsy in the diagnosis of interstitial lung disease. Chest 1993; 103: 765-770.

7. Carnochan FM, Walker WS, Cameron EWJ. Efficacy of video assisted thoracoscopic lung biopsy: a historical comparison with open lung biopsy. Thorax 1994; 49: 361-363.

8. Bove P, Ranger W, Pursel S, Glover J, Bove K, Bendick P. Evaluation of outcome following open lung biopsy. The American Surgeon 1994; 60: 564-570.

9. Molin LJ, Steinberg JB, Lanza LA. VATS increases costs in patients undergoing lung biopsy for interstitial lung disease. Ann Thorac Surg 1994; 58: 1595-1598.

10. Ravini M, Ferraro G, Barbieri B, Colombo P, Rizzato G. Changing strategies of lung biopsy in diffuse lung diseases: the impact of video-assisted thoracoscopy. Eur Respir J (in press).

11. Hunninghake GW, Crystal RG. Pulmonary sarcoidosis: a disorder mediated by excess helper T-lymphocyte activity at sites of disease activity. N Engl J Med 1981; 305: 429434.

12. Costabel U, Zaiss AW, Guzman J. Sensitivity and specificity of BAL findings in sarcoidosis. Sarcoidosis 1992; 9 (Suppl. 1): 211-214.

13. Winterbauer RH, Lammert J, Selland M, Wu R, Corley D, Springmeyer. Bronchoalveolar lavage cell populations in the diagnosis of sarcoidosis. Chest 1993; 104: 352-361.

14. Thomeer M, Demedts M. Predictive value of CD4/CD8 ratio in bronchoalveolar lavage in the diagnosis of sarcoidosis (abstract). Sarcoidosis Vasc and Diffuse Lung Dis 1997; 14 (Suppl. 1) 36.

15. Kantrow SP, Meyer KC, Kidd P, Raghu G. The CD4/CD8 ratio in BAL fluid is highly variable in sarcoidosis. Eur Respir J 1997; 10: 2716-2721.

16. Leonard C, Tormey VJ, O'Keane C, Burke CM. Bronchoscopic diagnosis of sarcoidosis. Eur Respir J 1997; 10: 2722-2724. 